\title{
Awareness and Performance of Paramedics in Preventing toward Middle East Respiratory Syndrome Coronavirus Infection
}

\author{
Myeong- Jeong Chae' ${ }^{1}$, Jong-Geun Yun ${ }^{2}$, Boyoung Kim ${ }^{3}$ \\ ${ }^{1}$ Assistant Professor, Department of Nursing, Gwangju Women's University, Gwang-ju, Korea, ${ }^{2}$ Assistant Professor, \\ Department of Emergency Medical Services Honam University, Gwang-ju, Korea, ${ }^{3}$ Associate Professor, College of \\ Nursing, Gyeongsang National University, South Korea
}

\begin{abstract}
The purpose of this study is to examine the knowledge of Middle East Respiratory Syndrome Coronavirus (MERS), wearing of protective equipment and the performance in preventing MERS infection among paramedics and analyze correlations. The survey was conducted on 96 active paramedics in K city, South Korea. The data were analyzedusing the SPSS 21.0 programusing descriptive statistics, t-test, one-way ANOVA, Scheffe test and Pearson's correlation coefficient.

Through this analysis, it was found that higher knowledge of MERS resulted in higher scores in the actions to prevent infection. As such, it is necessary to raise the infection control performance of paramedics by reflecting strategies for 'preparedness' rather than 'response' in the training on MERS, which must focus on knowledge and infection prevention.
\end{abstract}

Keywords: Paramedics, MERS-coronavirus, knowledge, protective equipment, infection prevention.

\section{Introduction}

Middle East respiratory syndrome is a severe acute respiratory infection caused by the MERS ${ }^{[1]}$. Since the first confirmed case was reported in Saudi Arabia in 2012, cases have been reported around the world and the death toll has been steadily increasing ${ }^{[2]}$. In fact, it continues to occur in Korea, as there was a suspected case of MERS in September 2018 ${ }^{[3]}$. Similar to SARS, which hit Asia in 2003, there is still no cure or vaccine for the disease, which is accompanied by kidney failure along

\footnotetext{
Corresponding Author:

Boyoung Kim

$\mathrm{RN}, \mathrm{PhD}$, PMHNP, Associate Professor, College of

Nursing, Gyeoungsang Institute of Health Science,

Gyeongsang National University, Korea, 816-15 Jinju-

daero, Jinju, 52727, Gyeongnam, South Korea

Phone: $+82-55-772-8248$

Fax : +82-55-772-8222

e-mail: bkimjhu@gmail.com
}

with symptoms of severe acute respiratory diseases such as high fever, coughing and difficulty in breathing ${ }^{[4]}$.

Looking back at the outbreak of MERS in Korea in 2015, the system overseeing each component of the emergency medical system did not function properly during the outbreak and spread of MERS ${ }^{[4]}$. This is because essential guidelines were missing and even the guidelines available were difficult to apply in the field ${ }^{[5]}$. In addition, the infections were concentrated in medical environments and were highly prevalent among health and medical care workers ${ }^{[6]}$. In particular, as paramedics have the most direct contact with active patients with infectious diseases before hospital treatment, they can be exposed to infectious diseases through contact with patients ${ }^{[7]}$. For this reason, paramedics need to perform effective infection control actions to minimize their infections related to emergency medical care. In addition, they must conduct activities to prevent the spread of microorganisms in order to prevent exogenous healthcare-associated infections ${ }^{[5]}$. Actions to prevent infection include hand hygiene, personal hygiene and 
aseptic technique, invasive instrument management, wearing and removing protective equipment, disinfection and sterilization, quarantine and environmental management activities performed by paramedics ${ }^{[8]}$.

Monitoring and reporting the outbreak of infections are the starting point and backbone of infection control projects $^{7}$ and these projects can be properly carried out by accurately identifying infection control problems inherent to paramedics. In addition, effective infection control can be achieved by monitoring the measures taken to address the problems and the results of education through continuous infection monitoring activities ${ }^{[9]}$. However, there is a lack of research and education on infection control for paramedics. Although the safety management SOP (Standard Operating Procedures) standard for paramedics has been prepared and enacted to protect paramedics from infections and the importance of paramedic safety is being emphasized ${ }^{[10-11]}$, studies in Korea on infection control by paramedics before hospital treatment have only been performed by Yoon and Jung ${ }^{[12]}$. As such, it is necessary to present the proper direction for infection control based on the reality of paramedics in Korea.

Therefore, the purpose of this study is to provide a basic data on infection control by examining the relationship between the knowledge of MERS, wearing protective equipment and the performance level of infection prevention for paramedics who are susceptible to infections at emergency sites.

\section{Method}

Study Participants and Data Collection: The participants in this study were selected from those who understood its purpose and objective and agreed to voluntarily participate in the study among active firefighting officers who entered the fire academy in K-area for retraining. To protect the participants, approval for this study was obtained from the university's bioethics review committee. We explained to the participants that the data will be only used for research in addition to their contribution to the research and the details and method of research. We also informed them that they could withdraw from the study and explained the anonymity and confidentiality of the data and that all data will be destroyed after the study. We considered the ethical aspect for the participants by receiving written consent to participate in the study. We distributed 100 questionnaires considering the recovery rate and collected 96 questionnaires for the final analysis.
Research Variables: Korean tools to measure the performance of MERS knowledge and actions to prevent infection have not yet been developed and the infection control protocol in Korea clinically applies the protocol developed by the Centers for Disease Control and Prevention $(\mathrm{CDC})^{[3]}$. Therefore, this study developed a questionnaire on MERS knowledge, wearing protective equipment and infection prevention performance after consulting with a licensed professor of emergency medicine based on the infection control guidelines for paramedics ${ }^{[13]}$, materials presented by the Korea Centers for Disease Control and Prevention, training materials for Korean paramedics to prevent MERS infection and MERS response integrated administrative guidelines ${ }^{14}$. The questionnaire has a total of 30 yes/no questions, including 10 questions about knowledge, 5 questions for protective equipment and 15 questions for infection prevention performance.

Data analysis: The data collected in this study were analyzed using the SPSS 21.0 program descriptive statistics, t-test, one-way ANOVA, Scheffe test and Pearson's correlation coefficient.

\section{Result and Discussion}

General Characteristics: The general characteristics of the paramedics are shown in Table 1 below. Out of 96 paramedics, $84(87.5 \%)$ were male and $12(12.5 \%)$ were female, so there about 3 times more male paramedics than female paramedics. In terms of age, 53 paramedics were under $29(55.2 \%)$ and 43 were over 30 years old (44.8\%). In terms of education, the paramedics consisted of 8 high-school graduates $(8.3 \%), 36$ university graduates $(37.5 \%)$ and 52 college graduates $(54.2 \%)$.

Performance of wearing protective equipment: The participants' performance of wearing protective equipment against MERS is shown in $<$ Table $3>$. The items with the highest performance level were $95.8 \%$ for 'wear N95 masks' and 'wear gloves,' while the item with the lowest performance level was $91.7 \%$ for 'wear gowns (protective coveralls) that cover the sleeves.' The use of protective equipment is the last defense to prevent harmful factors from entering the human body and here the average performance rate was high, at $93.7 \%$.

Knowledge of MERS, wearing protective equipment and preventing infection according to general characteristics: The differences in knowledge of MERS, wearing protective equipment and preventing 
infection according to the general characteristics of the paramedics are shown in $<$ Table $3>$. There were no differences according to gender, age and educational background. There was a statistically significant difference in wearing protective equipment according to the position or rank $(\mathrm{F}=3.621, p<.031)$ and the postanalysis showed that fire captains scored higher than fire fighters.

Relationship between Variables: The correlations between the participants' knowledge of MERS, wearing protective equipment and preventing infection are shown in $<$ Table $4>$. The higher the knowledge of MERS, the higher the score for actions to prevent infection $(\mathrm{r}=.216$, $p<.034)$.

The MERS outbreak is a historical event which brought about a paradigm shift in Korea's national infectious disease prevention and response system ${ }^{[9]}$. The outbreak of MERS was prevalent in medical institutions and between humans ${ }^{4}$. In Korea, the spread of the MERS outbreak was attributed to a complex interplay of various individual factors including the lack of awareness by medical professionals, the culture of family nursing, the health care delivery system and the initial response system of the government. We will not be able to achieve the desired results when faced with a complicated crisis such as MERS with conventional medical knowledge alone. Therefore, the first step in building an effective infection prevention system against infectious diseases such as MERS is to enhance the awareness and related knowledge through education in infection control guidelines based on the requirements of occupational roles and responsibilities, such as paramedics who experience direct contact with patients $^{[15]}$.

In addition, in order to be able to operate an effective infection prevention system in the event of a pandemic, regardless of the prevalence of MERS epidemic, it is necessary to develop an effective educational program for specific guidelines in all areas regarding the prevention and management of radio waves and to introduce and apply it in paramedics practice ${ }^{[16]}$. For example, measures should be developed to effectively train workers in MERS quarantine guidelines by implementing simulationbased training for MERS outbreak and regular training for wearing protective equipment, case management for MERS management according to MERS quarantine guidelines regularly updated by the Korea Centers for Disease Control ${ }^{[17]}$. These changes are essential for the education of occupational groups in direct contact with patients by preparing strategies related to preparedness rather than response.

The limitations of this study include it having a design, possible reporting bias and being conducted in a single city in Korea with a small sample size limited to a single profession. Based on the results of this study, the following suggestions are made. Since the results of this study are limited to the paramedics in Korea, follow-up research should be conducted in other countries to allow for greater comparison.

Table 1. Subjects' General Characteristics ( $\mathrm{N}=96)$

\begin{tabular}{|l|l|c|c|}
\hline General characteristics & Sort & Number of people & Percent (\%) \\
\hline \multirow{3}{*}{ Gender } & Male & 84 & 87.5 \\
\cline { 2 - 4 } & Female & 12 & 12.5 \\
\hline \multirow{3}{*}{ Age } & $\leq 29$ & 53 & 55.2 \\
\cline { 2 - 4 } & $\geq 30$ & 43 & 44.8 \\
\hline \multirow{3}{*}{ Education } & High-school graduate & 8 & 8.3 \\
\cline { 2 - 4 } & College graduate & 52 & 54.2 \\
\cline { 2 - 4 } & University graduate & 36 & 37.5 \\
\hline
\end{tabular}


Table 2. Performance of wearing MERS protective equipment ( $N=96)$

\begin{tabular}{|l|c|}
\hline Question & Performance level \\
\hline Wear N95 masks & 95.8 \\
\hline Wear gloves & 95.8 \\
\hline Wear gowns (protective coveralls) that cover the sleeves & 91.7 \\
\hline If necessary, wear goggles (when suspected patients cough, etc.) & 92.7 \\
\hline If necessary, wear face masks (when suspected patients cough, etc.) & 92.7 \\
\hline Total & $\mathbf{9 3 . 7}$ \\
\hline
\end{tabular}

Table 3. Knowledge of MERS, wearing protective equipment and preventing infection according to the general characteristics $(\mathbf{N}=96)$

\begin{tabular}{|c|c|c|c|c|c|c|c|}
\hline \multirow{2}{*}{$\begin{array}{l}\text { General } \\
\text { characteristics }\end{array}$} & \multirow{2}{*}{ Type } & \multicolumn{2}{|c|}{ Knowledge } & \multicolumn{2}{|c|}{ Wearing protective equipment } & \multicolumn{2}{|c|}{ Prevention } \\
\hline & & $\mathbf{M} \pm \mathbf{S D}$ & $\mathbf{t} / \mathbf{F}(\mathbf{p})$ & $\mathbf{M} \pm \mathbf{S D}$ & $\mathbf{t} / \mathbf{F}(\mathbf{p})$ & $\mathbf{M} \pm \mathbf{S D}$ & $t / F(p)$ \\
\hline \multirow{2}{*}{ Gender } & Male & $10.95 \pm 1.18$ & \multirow{2}{*}{$\begin{array}{c}.331 \\
(.741)\end{array}$} & $15.29 \pm 0.67$ & \multirow{2}{*}{$\begin{array}{c}.585 \\
(.560)\end{array}$} & $5.31 \pm 0.88$ & \multirow{2}{*}{$\begin{array}{l}-.090 \\
(.928)\end{array}$} \\
\hline & Female & $10.83 \pm 1.03$ & & $15.17 \pm 0.58$ & & $5.33 \pm 0.65$ & \\
\hline \multirow{2}{*}{ Age } & Under 29 & $9.23 \pm 2.64$ & \multirow{2}{*}{$\begin{array}{r}-1.090 \\
(.277)\end{array}$} & $60.70 \pm 6.78$ & \multirow{2}{*}{$\begin{array}{l}-.585 \\
(.559)\end{array}$} & $61.54 \pm 7.01$ & \multirow{2}{*}{$\begin{array}{l}-.232 \\
(.817)\end{array}$} \\
\hline & Over 30 & $9.69 \pm 1.74$ & & $60.02 \pm 6.72$ & & $61.84 \pm 9.16$ & \\
\hline \multirow{3}{*}{ Education } & $\begin{array}{l}\text { High-school } \\
\text { graduate }\end{array}$ & $11.50 \pm 1.07$ & \multirow{3}{*}{$\begin{array}{l}2.555 \\
(.083)\end{array}$} & $15.63 \pm 1.41$ & \multirow{3}{*}{$\begin{array}{l}1.286 \\
(.281)\end{array}$} & $5.88 \pm 1.46$ & \multirow{3}{*}{$\begin{array}{l}2.689 \\
(.073\end{array}$} \\
\hline & College graduate & $10.71 \pm 1.11$ & & $15.23 \pm 0.58$ & & $5.17 \pm 0.58$ & \\
\hline & $\begin{array}{l}\text { University } \\
\text { graduate }\end{array}$ & $11.14 \pm 1.20$ & & $15.25 \pm 0.50$ & & $5.39 \pm 0.96$ & \\
\hline \multirow{3}{*}{ Position (Rank) } & Fire fighters & $11.00 \pm 1.12$ & \multirow{3}{*}{$\begin{array}{c}.603 \\
(.550)\end{array}$} & $15.21 \pm 0.50$ & \multirow{3}{*}{$\begin{array}{l}3.621 \\
(.031) \\
(a<b)\end{array}$} & $5.25 \pm 0.59$ & \multirow{3}{*}{$\begin{array}{c}.174 \\
(.841)\end{array}$} \\
\hline & $\begin{array}{l}\text { Above fire } \\
\text { captain }\end{array}$ & $11.50 \pm 0.71$ & & $16.50 \pm 0.71$ & & $5.50 \pm 0.71$ & \\
\hline & Other & $10.80 \pm 1.10$ & & $15.27 \pm 0.72$ & & $5.33 \pm 0.84$ & \\
\hline
\end{tabular}

Table 4. Relationship between the knowledge of MERS, wearing protective equipment and preventing infection ( $\mathbf{N}=96)$

\begin{tabular}{|l|c|c|}
\hline Category & Knowledge $\mathbf{r}(\boldsymbol{p})$ & Actions to prevent infection $\mathbf{r}(\boldsymbol{p})$ \\
\hline Preventing infection & $.216^{*}(.034)$ & \\
\hline Wearing protective equipment & $.063(.543)$ & $-.021(.837)$ \\
\hline
\end{tabular}

$* \mathrm{p}<.05$

\section{Conclusion}

Our study shows that as paramedics have more knowledge of MERS, they had higher scores for preventing infection. Therefore, it is necessary to build an effective infection prevention system for infectious diseases by reflecting our knowledge of MERS and specific details on preventing infection in the education for occupational groups in direct contact with patients.

Ethical Clearance: Taken from Gwangju Women's University.
Source of Funding: Self

\section{Conflict of Interest: NA}

\section{Reference}

1. Korea centers for disease control and prevention. Daily report of MERS. [internet]. available at: http://www.mers.go.kr/mers/html/jsp/main.jsp. Retrieved 15 March 2018.

2. Newsway [internet]. available at: http:// www.newsway.co.kr/news/view?tp=1 \& 
$\mathrm{ud}=2015052021230338375$ Retrieved 19 August 2019.

3. Centers for disease control and prevention. About MERS. [internet]. Atlanta(GA): Centers for Disease Control and Prevention. available at: https://www. cdc.gov/coronavirus/mers/about/index/Retrieved 7 June 2017.

4. Suwantarat N, Apisarnthanarak A. Risks to healthcare workers with emerging diseases: Lessons from MERS-CoV, Ebola, SARS and avian flu. CurrOpin Infect Dis. 2015; 28(4):349-361.

5. National Health Insurance Service. Health in Magazine Theme story [internet]. Wonju, National Health Insurance Service. http://hi.nhis.or.kr/ magazin/120/html/sub_01.html. Retrieved 7 June 2018.

6. Banik GR., Khandaker G, Rashid H. Middle East respiratory syndrome coronavirus "ERS-CoV" current knowledge gaps. Paediatr Respir Rev. 2015; 16(3):197-202.

7. Choi JS, Kim JS. Factors influencing preventive behavior against middle east respiratory syndromecoronavirus among nursing students in south Korea. Nurse Educ Today. 2016; 40:168-172.

8. Ghobain MA, Aldrees T., Alenezi A, Alqaryan S, Aldabeeb D, Alotaib N, Aldhabib A, Alghalibi, S, Alhareth S. 2017. Perception and attitude of emergency room resident physicians toward Middle East respiratory syndrome outbreak. Emerg Med Int.2017; 1-4.

9. Park SM., Lee HJ, Choi JH, KimJH. Infection management for emergency rescue equipment. Korean J Emerg Med Serv. 2017;21(1):87-98.
10. Jeong, JY, Keong EK, Yun HY. Infection control awareness and practice for clothing management in 119 emergency medical technicians. Korean J Emerg Med Serv. 2014; 18(2):21-33

11. Jeonbuk Fire safety headquarters. Standard operation procedure(SOP) for disaster field. available at: http://www.sobang.kr/ board/view.sko?boardId=sobang_notice \& menuCd $=$ AA04001000000 \& dataSid $=9667$. Retrieved 26 August 2011.

12. Yun HW, Jung JY. A Study on the level of Recognition and Practices of 119 Emergency Medical Technician about Infection Control. Korean J Emerg Med Serv. 2008;12(3):99-113.

13. Korea paramedic safety management(SOP). available at: http://www.sobang.kr/board/ view.sko?boardId=sobang_notice \& menuCd=AA04001000000 \& dataSid $=9667$. Retrieved11 August 2019.

14. Ministry of health and welfare. 2015. [internet]. available at: https://www.mohw.go.kr/eng/ Retrieved 26 August 2019.

15. Kim SJ, Song RY. Knowledge and Practice of Middle East Respiratory Syndrome Isolation Precaution among Hospital Nurses. J Korean AcadFund Nurs. 2018; 25(1):46-57.

16. Kim, JY. Nurses' experience of Middle East respiratory syndrome patients care. J Korea AcadIndust coop Soc.2017; 18(10):185-196.

17. Hankyoreh 21 [internet]. available at: http://www. hani.co.kr/arti/society/society_general/862374. htmlRetrieved 7 June 2019. 